\title{
Characterization and Dissolution Study of Micellar Curcumin-Spray Dried Powder for Oral Delivery
}

This article was published in the following Dove Press journal:

International Journal of Nanomedicine

\author{
Nina Wijiani \\ Dewi Isadiartuti \\ M Agus Syamsur Rijal \\ Helmy Yusuf $(\mathbb{D}$
}

Department of Pharmaceutics, Universitas Airlangga, Surabaya, Indonesia

Correspondence: Helmy Yusuf Department of Pharmaceutics, Faculty of Pharmacy, Universitas Airlangga, Surabaya 60115 , Indonesia

Tel +62315933150

Fax +62 31 5935249

Email helmy-yusuf@ff.unair.ac.id
Introduction: Curcumin faces a major challenge in clinical use due to its poor aqueous solubility, which affects its bioavailability over oral use. The present study was carried out to overcome this problem.

Methods: An amorphous micellar curcumin-spray dried powder (MC-SDP) with selfassembled casein was prepared by the addition of sucrose as a protectant. The dry powder of curcumin-loaded micelles was obtained by a spray-drying technique in the presence of sucrose as a protectant. The MC-SDP in the form of dry powder was further developed into tablets to investigate the dissolution profile. The physical properties of preformed powder were characterized by differential thermal analysis (DTA), X-ray diffraction (XRD), and scanning electron microscopy (SEM). Quantitative analysis in the form of solutions was analyzed by high-performance liquid chromatography (HPLC).

Results: The physical properties demonstrated that MC-SDP varies from dented to smoother surfaces as a function of sucrose. Furthermore, melting transitions of curcumin in the form of MC-SDP were broadened in all sample mixtures, as observed in the DTA thermogram. The XRD spectra showed that the sharp and very intense peaks of single curcumin crystalline structure no longer existed in all MC-SDP forms, indicating that the mixtures were amorphous. Moreover, a further dissolution study of MC-SDP showed a significant increase of drug dissolved with the presence of sucrose, where $>80 \%$ of curcumin from MC-SDP was dissolved within $30 \mathrm{~min}$.

Conclusion: The study demonstrated the manufacture of micellar spray-dried powder that would contribute to the development of oral delivery of curcumin.

Keywords: micellar, amorphous, crystalline, solid dispersion, dissolution, powder

\section{Introduction}

Curcumin, also called diferuloylmethane, is the largest active compound contained in turmeric rhizome (Curcuma longa). The intensified investigations on curcumin are fundamentally due to the finding that curcumin exhibits several therapeutic effects, including antioxidant, anti-inflammatory, anticancer, anti-Parkinson's, and anti-Alzheimer's effects. $^{1-4}$

To date, curcumin faces a major challenge for clinical use due to its poor aqueous solubility, which massively affects its bioavailability over oral use. ${ }^{5}$ Alkaline hydrolysis followed by molecular fragmentation was reported as the major cause of curcumin rapid degradation. ${ }^{6}$ Strategies have been employed to improve the aqueous solubility as well as bioavailability of curcumin by using several delivery systems including nanoparticles, liposomes, solid lipid nanoparticles, complex inclusions, and

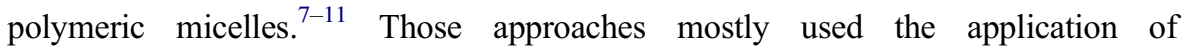


nanotechnology with the use of polymers. ${ }^{12}$ Furthermore, an enhanced bioavailability of curcuminoids in the brain was achieved following intranasal administration by using polymeric nanoparticles. ${ }^{13}$ Many polymers have been widely applied to improve the solubility of poorly watersoluble drugs. The use of biodegradable polymers has had excellent results in the development of drug delivery systems for mucosal delivery including oral, intranasal, and pulmonary mechanisms. ${ }^{14-16}$ Attractive designs of delivery systems for curcumin have been rapidly developed in this area including the use of zein-hyaluronan-based nanoparticles. ${ }^{17,18}$

Among the above-mentioned delivery systems, polymeric micelles have recently attracted much attention in overcoming solubility-related problems of curcumin. ${ }^{14,19}$ Polymeric micelles, most of them are surfactants, consist of amphiphilic block copolymers that spontaneously assembled into nano-sized structures in aqueous solution. The micellar structure consists of two parts, called the core and the corona. ${ }^{20}$ Hydrophobic drugs can be entrapped into their hydrophobic core. In contrast, for reversed micelles, the core is the hydrophilic part. This system can last longer in blood circulation and increase the halflife in the systemic circulation. ${ }^{21}$ Furthermore, it has been reported that the encapsulation of curcumin in micelles segregates them from the alkaline environment and prevents them from hydrolysis. ${ }^{19}$

Most micellar surfactants that have been investigated in previous decades used synthetic surfactants including sodium caseinate, tetradecyltrimethylammonium chloride (TTAC), or hexadecyltrimethylammonium bromide (CTAB). ${ }^{22,23}$ More recently, natural micellar-forming polymers such as casein have attracted interest in the development of micelle delivery systems. ${ }^{24,25}$ As a natural biodegradable polymer, casein is classified as generally recognized as safe (GRAS), and is commonly known as safe, biodegradable, and does not affect the immune responses. ${ }^{26}$ Many casein-based pharmaceutical products are in clinical evaluation. ${ }^{27}$ Immense investigations have discovered curcumin's instability in a liquid formulation. $^{28,29}$ Therefore, solid formulations are of particular interest. ${ }^{30,31}$

In the present study, further processing was carried out to transform micellar curcumin into a solid dispersion powder. The developed formulations were expected to enhance the solubility and bioavailability upon oral administration. At the same time, the dry product could ease the handling and improve stability. The physicochemical characteristics of micellar curcuminspray dried powder (MC-SDP) were investigated in terms of their morphology using scanning electron microscopy (SEM); the thermal properties were investigated using differential thermal analysis (DTA); and the crystallography structure was investigated using X-ray diffractometry (XRD). Also, a dissolution experiment was carried out, in which the dissolved curcumin concentration was measured and analyzed using highperformance liquid chromatography (HPLC).

\section{Materials and Methods \\ Materials}

Curcumin (CUR), casein (CAS), sucrose (SUC), and D- $\alpha$ tocopherol polyethylene glycol-1000 succinate (TPGS) were purchased from Sigma-Aldrich (Singapore). Ethanol was purchased from E. Merck (Singapore).

\section{Preparation of Micellar Curcumin-Spray Dried Powder}

A solution of casein was prepared at $\mathrm{pH} 7$ in a concentration of $3 \mathrm{mg} / \mathrm{mL}$ (much higher than its critical micellar concentration). TPGS was dissolved in aquadest and added to the casein solution. Sucrose was dissolved in aquadest and added to the mixture of casein and TPGS to make a total volume of $250 \mathrm{~mL}$. Curcumin (75 mg) was dissolved in ethanol q.s. and then added dropwise to the mixture under constant stirring $(850 \mathrm{rpm})$ for $5 \mathrm{~h}$. The formed micelles were further spray dried using a Buchi B-290 Mini-Spray Dryer (Flawil, Switzerland) equipped with a high-performance cyclone. The spray-drying conditions were as follows: inlet temperature of $150^{\circ} \mathrm{C}$, outlet temperature of $90^{\circ} \mathrm{C}$, spraying pressure of 5.0-5.8 mbar, feed flow of $5 \mathrm{~mL} / \mathrm{min}$, airflow rate of $320 \mathrm{~L} / \mathrm{h}$ and aspiration air of $90 \%$. The obtained MC-SDP was collected in a tight container and stored in a desiccator at $25^{\circ} \mathrm{C}$ until further analysis. Four different formulations of MC-SDP were prepared to make up a weight ratio (\%) of CAS:TPGS:SUC as follows: 17:1:0 (F1); 17:1:25 (F2); 42.5:1:0 (F3) and 42.5:1:25 (F4).

\section{Determination of Drying Yield (\%)}

The percentage of drying yield was calculated from the total dry powder of MC-SDP collected following the spray-drying process in proportion to the initial amount of materials in the formula. Yield percentage was obtained by the following equation: 


$$
\% \text { Yield }=\frac{\text { total weight of dry powder }}{\text { total weight of initial formula }} \times 100 \%
$$

\section{Morphology Analysis Using Scanning Electron Microscopy}

The morphology of the dry product of MC-SDP was analyzed using SEM. Portions of the dry powder were scattered and glued onto $25 \mathrm{~mm}$ diameter plates, and further attached to specimen mounts. Samples were further sputter-coated with a layer of gold for $5 \mathrm{~nm}$ in thickness (approximately for $180 \mathrm{~s}$ ) and examined with an electron microscope (Phenom, USA).

\section{Thermal Analysis Using Differential Thermal Analysis}

DTA instrument (Mettler Toledo FP 85, Switzerland) was used to detect the solid state of the dry product of MCSDP. Samples were placed in aluminum crucibles and the DTA analysis was performed at a heating rate of $10^{\circ} \mathrm{C} /$ min, covering temperature ranges from $30^{\circ} \mathrm{C}$ to $300^{\circ} \mathrm{C}$.

\section{Crystallography Analysis Using X-Ray Diffractometry}

The crystallography structure of the dry MC-SDP was examined by an X-Ray Diffraction Instrument (Phillips X'Pert PRO PAN Analytical, the Netherlands). Samples were inserted into the holder and surface leveled. Furthermore, the holder was inserted into the instrument.
The conditions of the analysis were set as follows: voltage $40 \mathrm{kV}$, current $25 \mathrm{~mA}$, angular range of $5-40^{\circ}(2 \theta)$, and scanning speed of $2 \% \mathrm{~min}$.

\section{Dissolution Study}

Thirty milligrams of MC-SDPs (equal to $1.0 \mathrm{mg}$ curcumin) were made into a mini-tablet using a single punch hydraulic press (Natoli Engineering Company, USA) with a force of $2000 \mathrm{~N}$. The tablets were added to $500 \mathrm{~mL} \mathrm{pH}$ 6.8 phosphate buffer. The temperature of the dissolution medium was set at $37^{\circ} \mathrm{C}$ and a stirring paddle was set at $100 \mathrm{rpm}$. Dissolved curcumin concentration as a function of time was sampled at 5, 10, 15, 20, 25 and 30 min time intervals and analyzed using the HPLC. The absorption peak at $421 \mathrm{~nm}$ was used to monitor the dissolved curcumin. All measurements were performed in triplicate.

\section{Results and Discussion Drying Yield (\%w/w)}

Figure 1 shows that incorporating sucrose in MC-SDP formulation (F2 and F4) substantially improved the yield rate in which F2 with $73.4 \pm 1.6 \%$ and F4 with a drying yield of $91.9 \pm 3.1 \%(\mathrm{w} / \mathrm{w})$. Excluding sucrose from formulations (F1 and F3) resulted in decreased drying yield of MC-SDP, where F1 with $64.4 \pm 0.6 \%$ and F3 with only 60.1 $\pm 1.7 \%$. The presence of sucrose as protectant produced a greater yield percentage, as it might increase the mass density of the powder and prevent deposition of material on the wall of the spray dryer chamber. Powder loss is

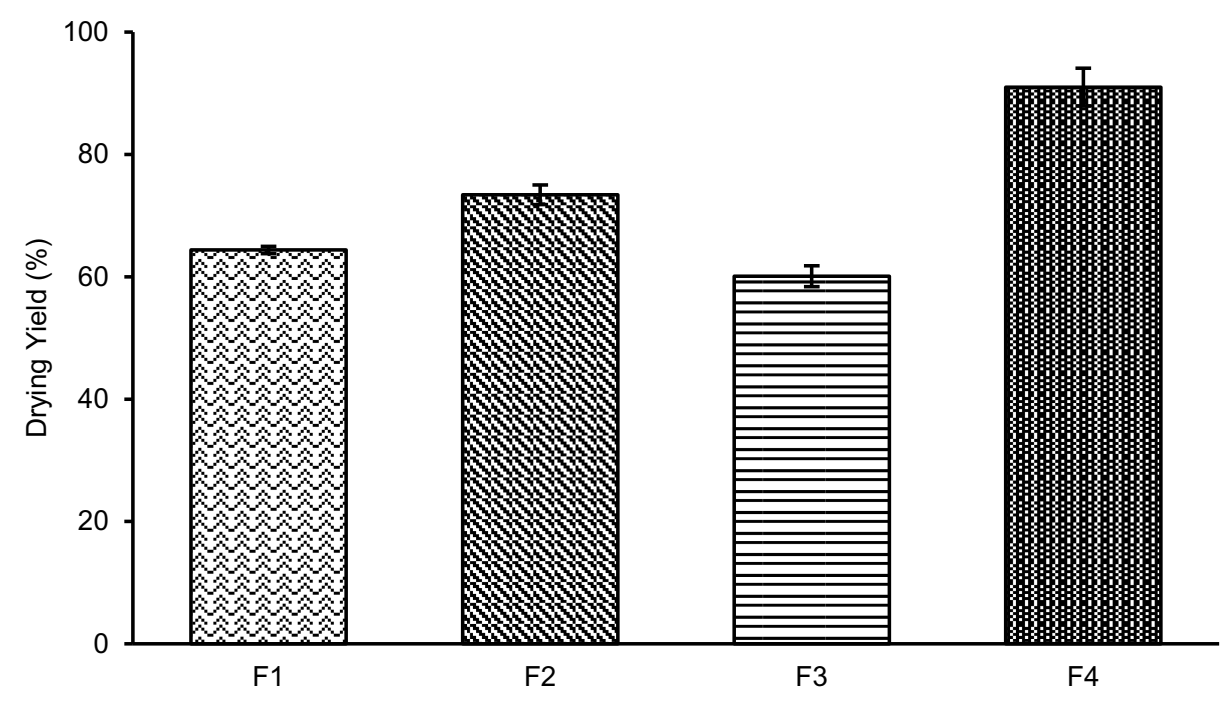

Figure I Drying yield percentage of MC-SDPs containing curcumin made at different percentage weight ratios of CAS:TPGS:SUC. Notes: (FI) 17:1:0; (F2) 17:1:25; (F3) 42.5:1:0; (F4) 42.5:1:25.

Abbreviations: MC-SDPs, micellar curcumin-spray-dried powders; CAS, casein; TPGS, D- $\alpha$-tocopherol polyethylene glycol-I 000 succinate; SUC, sucrose. 
generally caused by stickiness to the wall and the cyclone's poor efficiency in collecting fine particles. ${ }^{32,33}$

\section{Scanning Electron Microscopy}

The morphology of MC-SDP was examined by SEM and is presented in Figure 2. The surface morphology of MCSDP formulations without sucrose showed dented surfaces on some particles with a wrinkle-like structure (F1 and F3). This was slightly different from the MC-SDP that incorporated sucrose to the formulations (F2 and F4). Adding
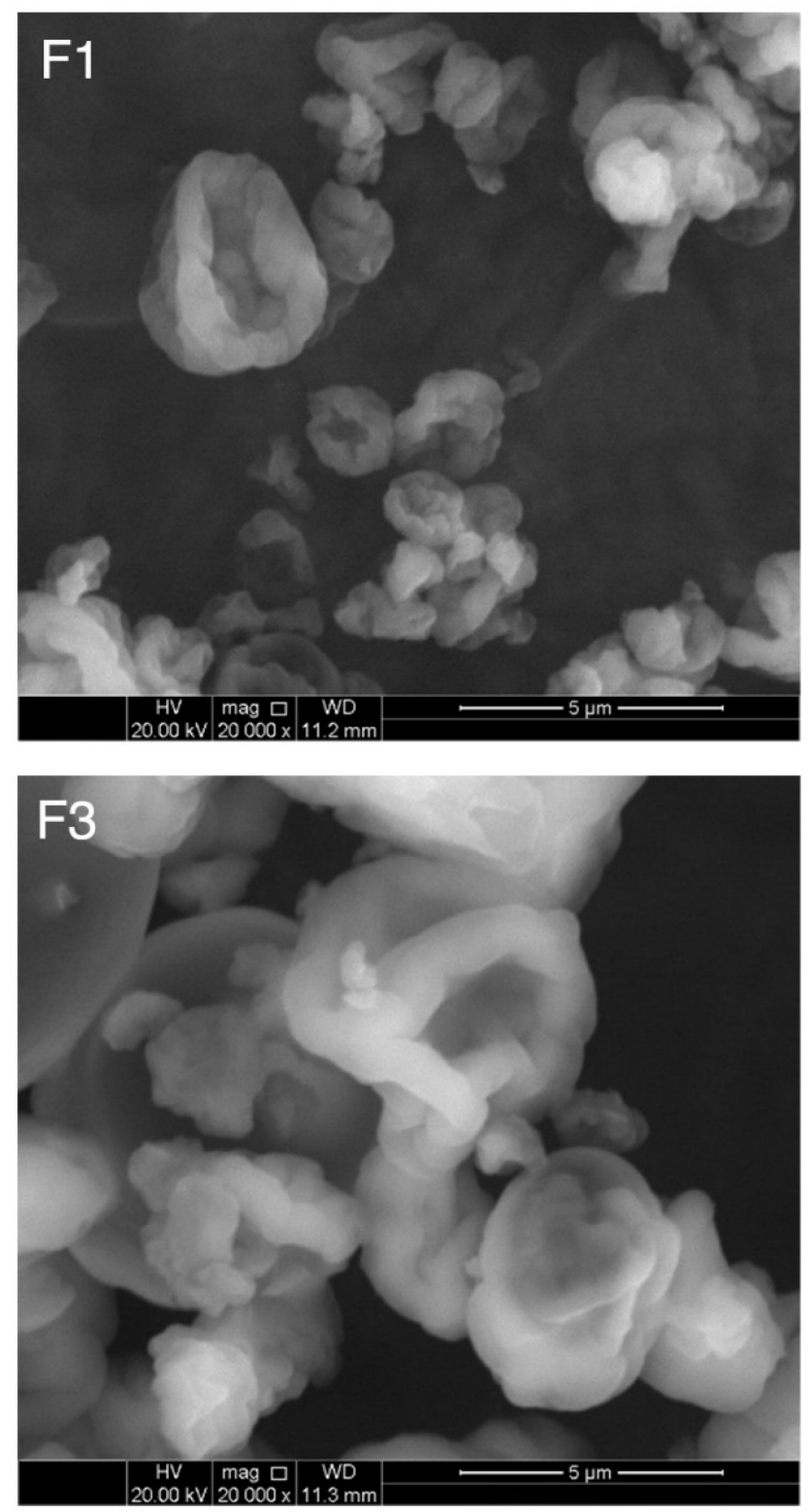

sucrose to MC-SDP formulations resulted in smoother surfaces in which the wrinkle-like structures were not observed. In this case, sucrose might be a determinant factor that could alter the surface morphology among formulations. The presence of hygroscopic sucrose might stimulate agglomeration with surrounding smaller particles since they easily adsorb moisture (F2 and F4). From the results, the obtained particles varied in shape and size, and the dented-surface particles were no longer found, compared to those in the MC-SDP formulations without sucrose.
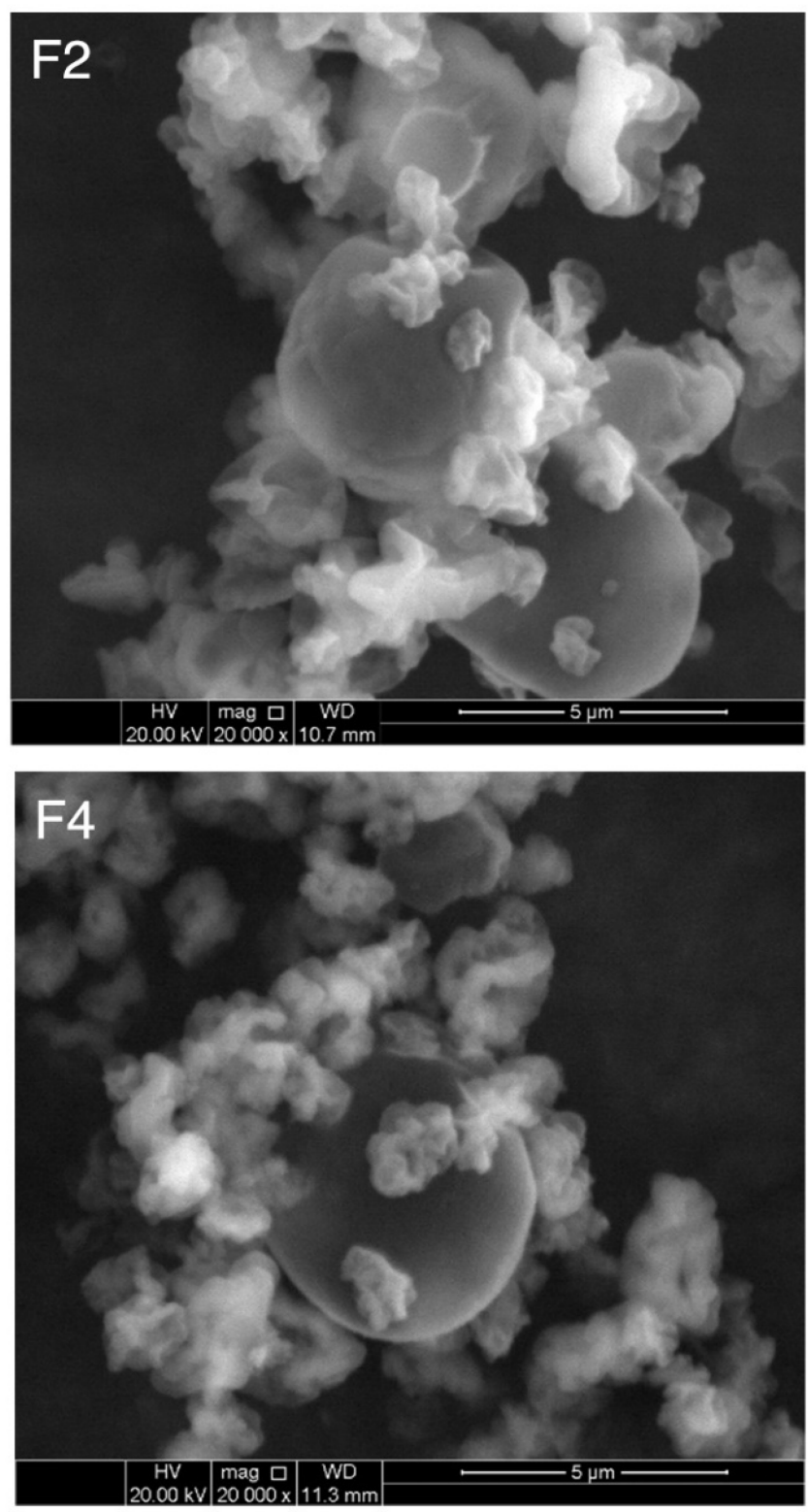

Figure 2 SEM photomicrograph of MC-SDPs containing curcumin made at different percentage weight ratios of CAS:TPGS:SUC. Notes: (FI) 17:I:0; (F2) 17:I:25; (F3) 42.5:I:0; (F4) 42.5:I:25.

Abbreviations: SEM, scanning electron microscopy; MC-SDPs, micellar curcumin-spray-dried powders; CAS, casein; TPGS, D- $\alpha$-tocopherol polyethylene glycol-I000 succinate; SUC, sucrose. 
The change of the surface particle morphology from the wrinkle-like structure (F1 and F3) to relatively smoother surfaces (F2 and F4) may highlight the benefit of including sucrose in spray-dried formulations. Other advantages are related to their ability to form interactions with casein during spray drying. Such interactions could preserve the native conformation of casein, and reduce the molecular mobility whilst transforming into solid. The use of sucrose was beneficial because such interactions are better formed with disaccharides compared to larger sugars. ${ }^{34}$ In the absence of sucrose, casein could not escape the drying interface and might be exposed to denaturation. Including sucrose in the formulation may reduce the chance of casein occurring on the particle surface. Hence, casein could be well surrounded by sucrose and prevented from heat-caused denaturation. ${ }^{35,36}$ Furthermore, sucrose may enhance the solid mass formation as it forms a macrostructure of solid particles during the spray-drying process, which could not be achieved by a formulation without sucrose. ${ }^{37}$

\section{Differential Thermal Analysis}

A DTA thermogram of single materials is presented in Figure 3. Single curcumin, casein, and sucrose exhibited endothermic peaks at a temperature range of $180-200^{\circ} \mathrm{C}$, whereas TPGS showed an endothermic peak at a much lower temperature of $37^{\circ} \mathrm{C}$
Figure 4 demonstrates broader endothermic peaks of MC-SDP formulations for $\mathrm{F} 1, \mathrm{~F} 2$, and $\mathrm{F} 3$ at $176.3^{\circ} \mathrm{C}$, $189.2^{\circ} \mathrm{C}$, and $192.7^{\circ} \mathrm{C}$, respectively. These peaks were not as sharp as the crystalline curcumin. Curcumin molecules might be homogeneously distributed and miscible with the other components in the mixture, indicated by a single and small endothermic peak. The presence of other molecules among curcumin molecules in the crystal lattice arrangement has weakened their hydrophobic bonding, resulting in smaller and broader peaks. Interestingly, there were no other endothermic peaks detected in the thermograms, indicating that a single-phase mixture was achieved. Furthermore, the smaller peaks might indicate the formation of a relatively more disordered crystalline state of the three MC-SDP formulations. This result is in agreement with a similar study investigating the thermal properties of curcumin in amorphous solid dispersions using hydroxypropyl methylcellulose (HPMC) as a polymeric matrix. ${ }^{38,39}$ The physical mixture also showed a single peak observed at a higher temperature $\left(220^{\circ} \mathrm{C}\right)$, which could be explained by the hot melting materials during the heating process, resulting in a homogenous dispersion. This was supported by further XRD data analysis that confirmed this suggestion.

However, this was not the case for F4, with a higher concentration of sucrose. The endothermic peak was sharper

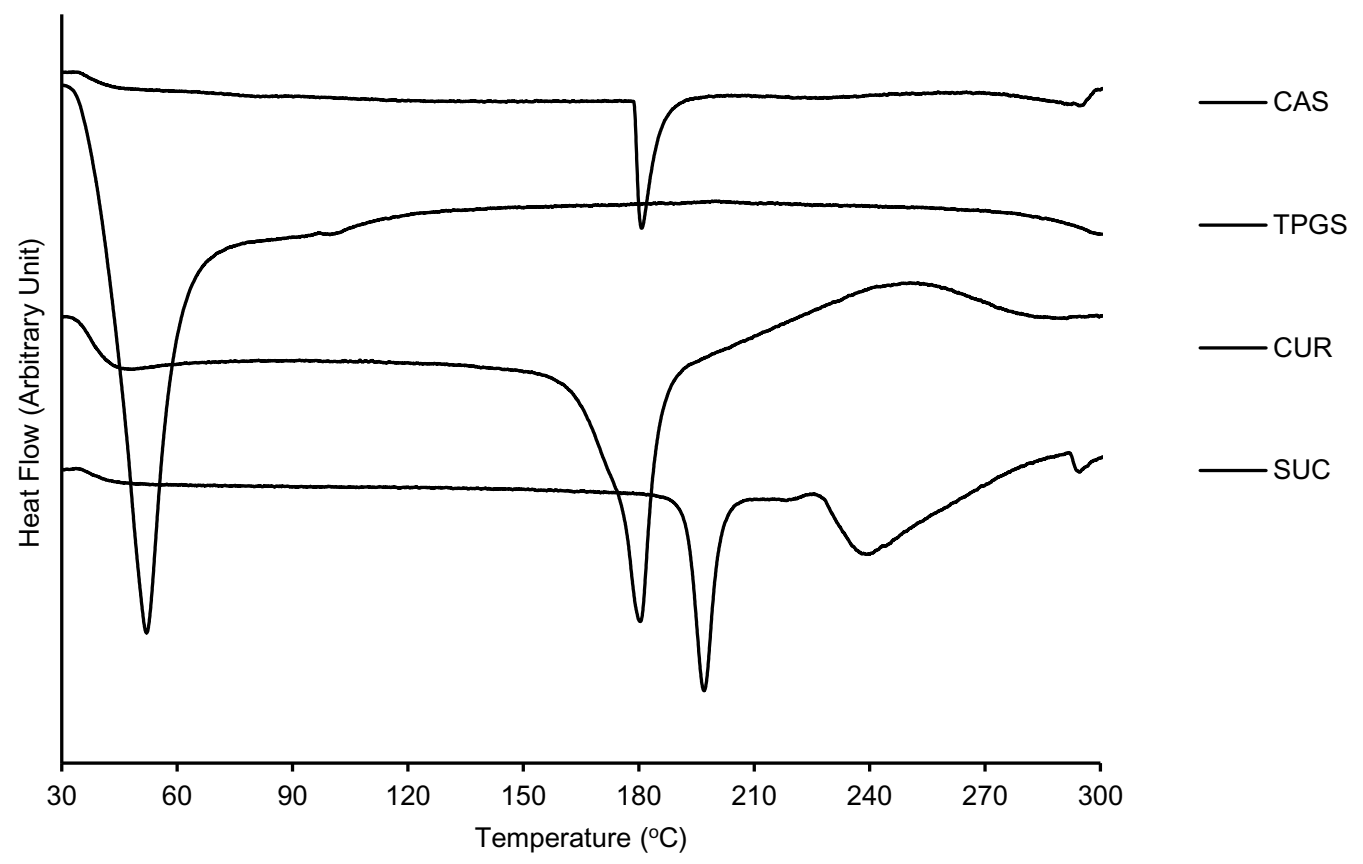

Figure 3 DTA thermograms of curcumin and single components used in the developed MC-SDP formulations.

Abbreviations: DTA, differential thermal analysis; MC-SDP, micellar curcumin-spray dried powder; CAS, casein; TPGS, D- $\alpha$-tocopherol polyethylene glycol-I000 succinate; CUR, curcumin; SUC, sucrose. 


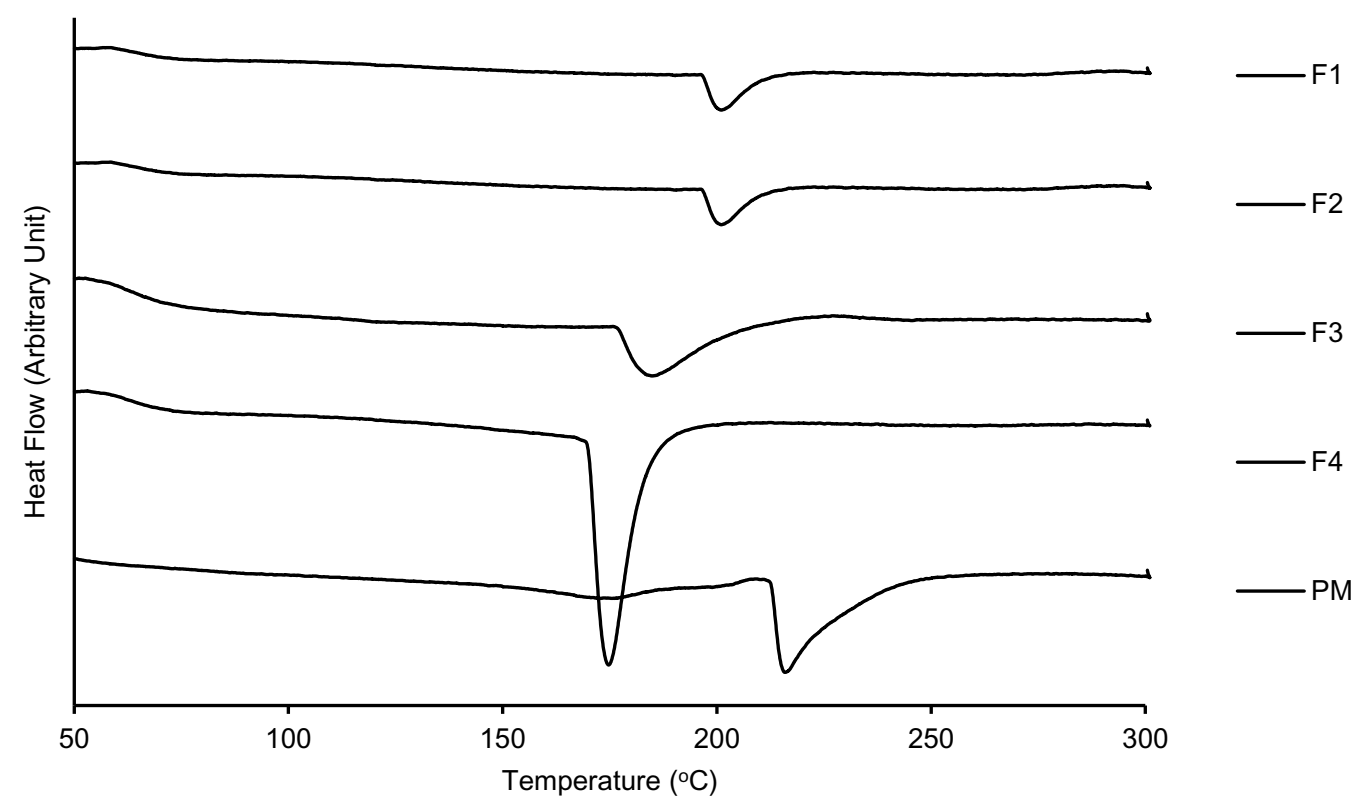

Figure 4 DTA thermograms of MC-SDPs containing curcumin made at different percentage weight ratios of CAS:TPGS:SUC and their physical mixture.

Notes: (FI) 17:1:0; (F2) 17:1:25; (F3) 42.5:1:0; (F4) 42.5:1:25; (PM) physical mixture of curcumin, casein, D- $\alpha$-tocopherol polyethylene glycol-1000 succinate, and sucrose. Abbreviations: DTA, differential thermal analysis; MC-SDPs, micellar curcumin-spray-dried powders; PM, physical mixture; CAS, casein; TPGS, D- $\alpha$-tocopherol polyethylene glycol-1000 succinate; CUR, curcumin; SUC, sucrose.

and larger. As sucrose is present at a much higher concentration, the mixture may be oversaturated. This oversaturated mixture might lead to stronger intermolecular interactions between curcumin and casein and sucrose, which may be responsible for the resulting thermal properties (Figure 4). Nevertheless, despite the fact that their intermolecular arrangement was more of a crystal-like structure, no single materials were phase separated from the mixtures. This was a strong indication that the employed matrix was able to stop drug molecules from being rearranged again and crystallized. The saturated sucrose in the mixture has been suggested to increase the tendency of internal interactions; however, the molecular dynamics were not sufficient to recrystallize. ${ }^{40,41}$ It can be confirmed that casein, as well as sucrose in the developed MC-SDP formulations, successfully prevented curcumin from recrystallization. Moreover, the employed ratio of polymeric casein and sucrose was sufficient to inhibit the crystallization of curcumin.

\section{X-Ray Diffraction Analysis}

Diffractogram patterns of all samples were determined using XRD analysis. This technique is one of the most robust techniques used to investigate the crystallographic structure of curcumin after encapsulation into micelles and spray drying into powder. The XRD data of single materials are shown in Figure 5. It was revealed that the diffraction patterns of curcumin, TPGS, and sucrose showed intense sharp peaks, implying the crystalline structure of the materials. Casein showed a different pattern, with broad patterns without any sharp peaks, indicating that the material was in a more disordered crystalline-structure or amorphous state.

Different patterns were observed for all MC-SDP formulations. All of these intensive and sharp peaks were no longer observed in MC-SDP formulations (Figure 6). Entrapped curcumin in the polymeric casein micelles, as well as sucrose and TPGS, were in a disordered crystalline or amorphous state. A more intensified interaction between curcumin with the hydrophobic groups of the casein molecules has caused the disappearance of the crystalline order of curcumin. ${ }^{42}$ This amorphous state, from the perspective of physicochemistry, is characterized by having higher energy, which is the cause of the increased water solubility. ${ }^{43}$ Moreover, the presence of sucrose seemed not to affect the crystallography structure of the developed formulations. There was no difference in terms of the diffraction pattern between formulations with and without sucrose, suggesting that the addition of sucrose did not alter the crystallography structure of MC-SDP. These results were in agreement with the DTA data, where casein, as well as sucrose, inhibited curcumin from recrystallization. These results were more convincing from the XRD data of the physical mixture. The crystalline peaks of all materials were apparent in the physical mixture. This data information 


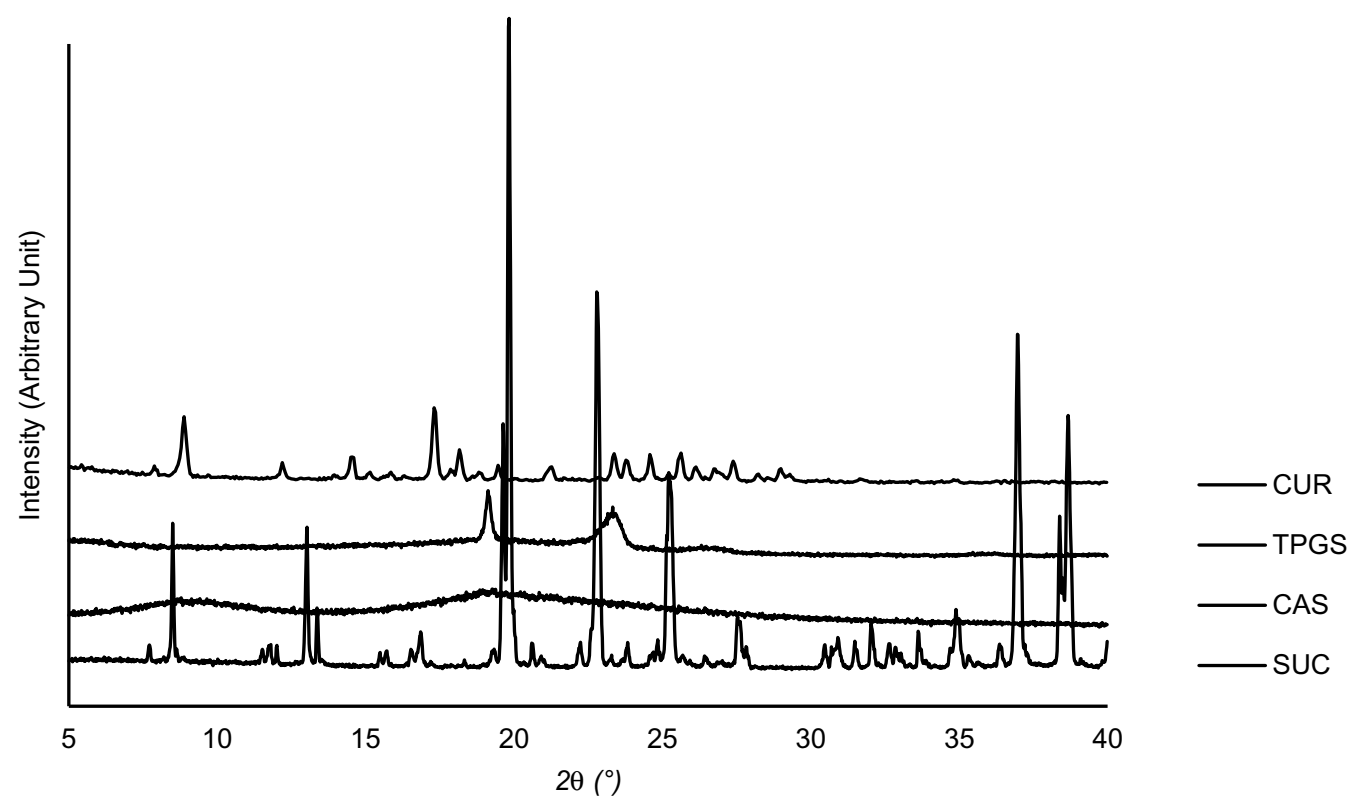

Figure 5 XRD pattern of curcumin and single components used in the developed MC-SDP formulations.

Abbreviations: XRD, x-ray diffractometry; MC-SDP, micellar curcumin-spray dried powder; CUR, curcumin; TPGS, D- $\alpha$-tocopherol polyethylene glycol-1000 succinate; CAS, casein; SUC, sucrose.

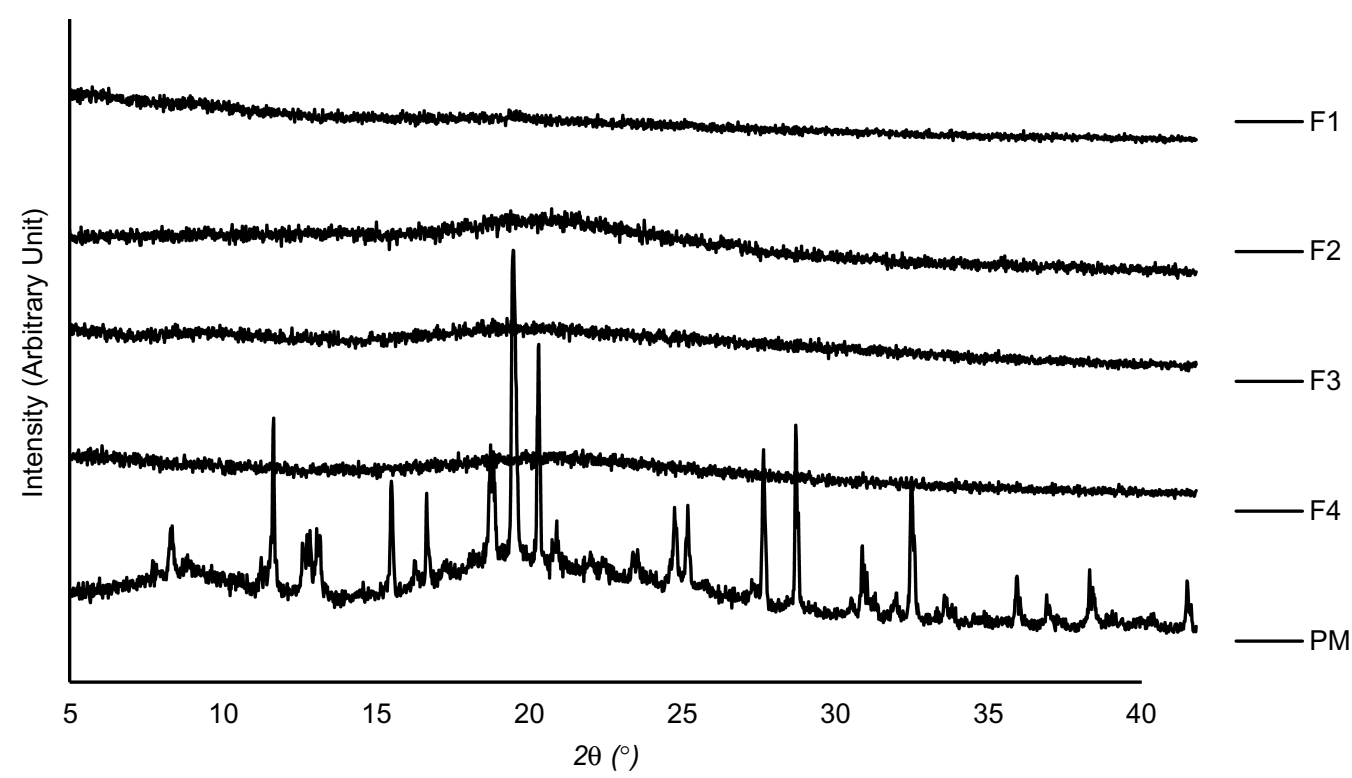

Figure 6 XRD patterns of MC-SDPs containing curcumin made at different percentage weight ratios of CAS:TPGS:SUC and their physical mixture.

Notes: (FI) 17:I:0; (F2) 17:I:25; (F3) 42.5:I:0; (F4) 42.5:1:25; (PM) physical mixture of curcumin, casein, D- $\alpha$-tocopherol polyethylene glycol-I000 succinate, and sucrose. Abbreviations: XRD, x-ray diffractometry; MC-SDPs, micellar curcumin-spray-dried powders; PM, physical mixture; CUR, curcumin; CAS, casein; TPGS, D- $\alpha$-tocopherol polyethylene glycol-1000 succinate; SUC, sucrose.

may highlight the lack of interactions of curcumin with other components in their solid state. XRD diffractograms of the physical mixture displayed the presence of peaks corresponding to all crystalline materials, thus confirming their existence which was not detected by DTA analysis where all materials seemed to be miscible and formed a single phase.

\section{Dissolution Study}

Figure 7 shows the dissolution profiles of MC-SDPs formulated with and without sucrose. The amount of MC-SDP added was based on the theoretical concentration of curcumin that is equivalent to $1 \mathrm{mg} \mathrm{w} / \mathrm{w}$. The percentage of dissolved curcumin achieved was noted between the different 


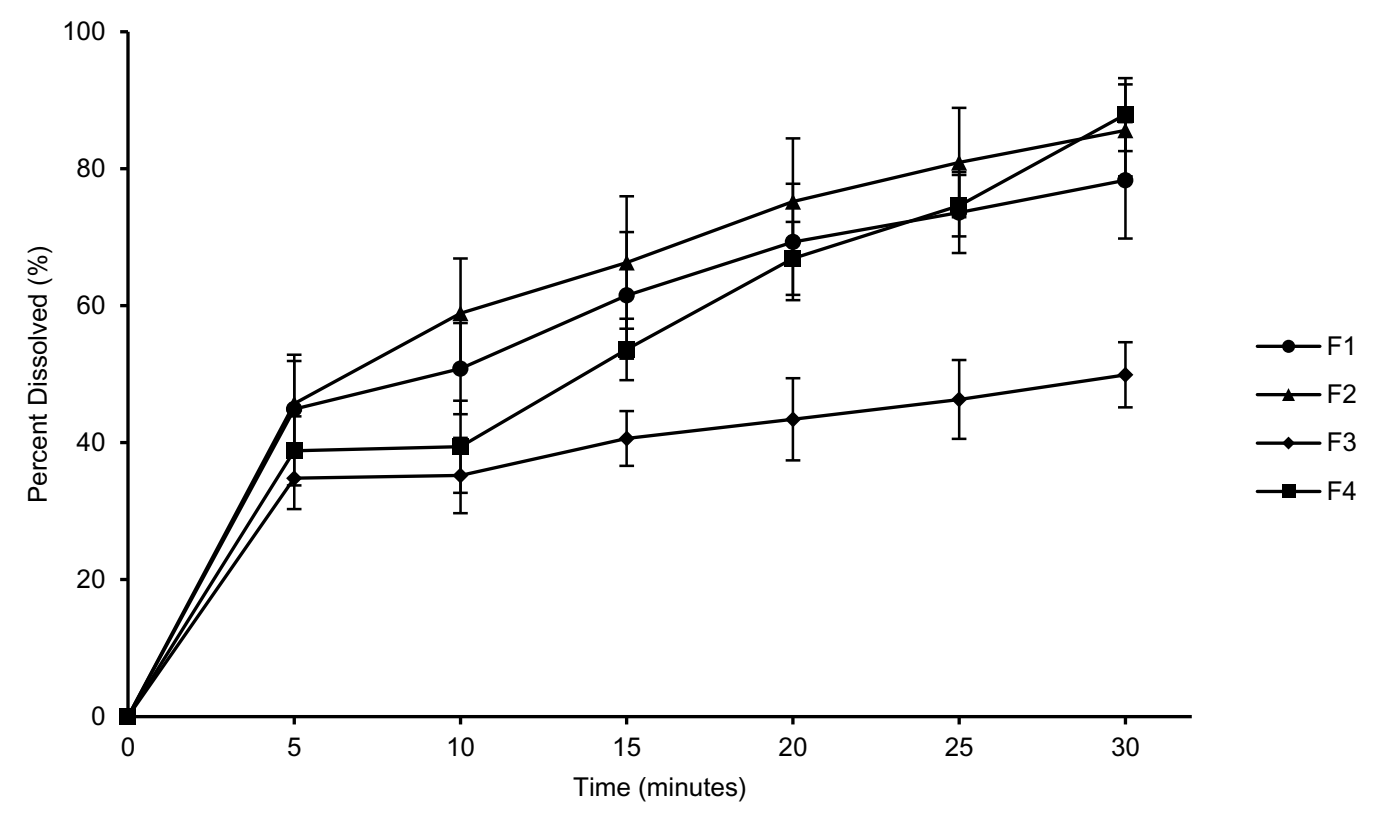

Figure 7 Dissolution profiles of curcumin formulated in MC-SDPs made at different percentage weight ratios of CAS:TPGS:SUC. Notes: (FI) 17:1:0; (F2) 17:1:25; (F3) 42.5:1:0; (F4) 42.5:1:25.

Abbreviations: MC-SDPs, micellar curcumin-spray-dried powders; CAS, casein; TPGS, D- $\alpha$-tocopherol polyethylene glycol-I000 succinate; SUC, sucrose.

formulations. Interestingly, MC-SDP with sucrose (F2 and F4) showed a higher percentage of dissolved curcumin than the corresponding MC-SDP without sucrose (F1 and F3). MC-SDP with the lowest amount of casein exhibited the lowest dissolution profile. The total dissolved curcumin achieved at the end of the experiment was $78.3 \pm 8.5 \%$; 85.6 $\pm 6.7 \% ; 49.9 \pm 4.8 \%$, and $87.9 \pm 5.3 \%$ for $\mathrm{F} 1, \mathrm{~F} 2, \mathrm{~F} 3$, and $\mathrm{F} 4$, respectively.

MC-SDP with sucrose (F2 and F4) offered a higher drug dissolution profile during the dissolution study. The dissolved curcumin achieved by MC-SDP formulations for F2 and $\mathrm{F} 4$ was $85.6 \%$ and $87.9 \%$, respectively, within $30 \mathrm{~min}$. On the other side, the percentage of dissolved curcumin for F1 and F3 only reached $78.3 \%$ and $49.9 \%$, respectively. It might be concluded from this that a higher amount of curcumin was present in an amorphous state with a combination matrix of casein and sucrose. A similar solid dispersion formulation which was prepared using sucrose alone or in combination with other surfactants or polymers has successfully maintained the amorphous state of both drug and the carrier and increased the solubility of the drug. ${ }^{44,45}$

Next, the determinant factor of the dissolution performance can be attributed to the impact of sucrose on the dissolution of curcumin. This phenomenon, ie, the presence of sugar molecules increasing the curcumin dissolution profile, very likely occurred through the formation of nanostructured molecular-level of curcumin/sucrose composites during spray-drying. A study reported that aggregated-nanostructures were formed whenever both sugar and poorly water-soluble drug molecules were present in aqueous media. It was revealed that different affinities among the drug and the aggregated nanostructures led to increased solubility, which may explain the enhanced dissolution of MC-SDP. ${ }^{46}$

\section{Conclusion}

Spray drying has been proven to be feasible in producing micellar-curcumin powder under proper manufacturing conditions with a suitable protectant, such as sucrose. Sucrose as protectant is essential for the development of MC-SDP formulation as it increases the yield rate. Also, the surface particle morphology was improved, with smoother surfaces by the presence of sucrose, as revealed by SEM images. The thermal analysis of the MC-SDP formulation suggested the disappearance of curcumin crystalline structure in the powder mixtures. Curcumin was well distributed and miscible with casein, sucrose, and TPGS, by creating a single phase and amorphous solid state as revealed by DTA and XRD analysis. Additionally, the MC-SDP formulations with sucrose enhanced the dissolution profile of the entrapped curcumin. The presence of sucrose has been proposed as the key element for this enhancement through the formation of a nanostructured composite between curcumin and sucrose. This study 
reveals a beneficial approach for possible oral delivery of MC-SDP formulation and may shed light on the further development of an oral dosage form.

\section{Acknowledgments}

This research was supported by the PTUPT Grant awarded by the Directorate General of Higher Education, Republic of Indonesia.

\section{Disclosure}

The authors report no conflicts of interest in this work.

\section{References}

1. Abrahams S, Haylett WL, Johnson G, Carr JA, Bardien S. Antioxidant effects of curcumin in models of neurodegeneration, ageing, oxidative and NITROSATIVE stress: a review. Neuroscience. 2019;406:1-21. doi:10.1016/j.neuroscience.2019.02.020

2. Fadus MC, Lau C, Bikhchandani J, Lynch HT. Curcumin: an age-old anti-inflammatory and anti-neoplastic agent. $J$ Tradit Complement Med. 2017;7(3):339-346. doi:10.1016/j.jtcme.2016.08.002

3. Panda AK, Chakraborty D, Sarkar I, Khan T, Sa G. New insights into therapeutic activity and anticancer properties of curcumin. $J$ Exp Pharmacol. 2017;9:31-45. doi:10.2147/JEP.S70568

4. Goozee K, Shah T, Sohrabi HR, et al. Examining the potential clinical value of curcumin in the prevention and diagnosis of alzheimer's disease. $B r \quad J$ Nutr. 2016;115(3):449-465. doi:10.1017/ S0007114515004687

5. Liu W, Zhai Y, Heng X, et al. Oral bioavailability of curcumin: problems and advancements. J Drug Target. 2016;24(8):694-702. doi:10.3109/1061186X.2016.1157883

6. Wang L, Lu N, Zhao L, et al. Characterization of stress degradation products of curcumin and its two derivatives by UPLC-DAD-MS/ MS. Arabian J Chem. 2016;12(8):3998-4005.

7. Ji H, Tang J, Li M, Ren J, Zheng N, Wu L. Curcumin-loaded solid lipid nanoparticles with Brij78 and TPGS improved in vivo oral bioavailability and in situ intestinal absorption of curcumin. Drug Deliv. 2016;23(2):459-470. doi:10.3109/10717544.2014.918677

8. Zhao Z, Xie M, Li Y, et al. Formation of curcumin nanoparticles via solution-enhanced dispersion by supercritical $\mathrm{CO} 2$. Int J Nanomedicine. 2015;10:3171-3181. doi:10.2147/IJN.S80434

9. Ding T, Li T, Wang Z, Li J. Curcumin liposomes interfere with quorum sensing system of aeromonas sobria and in silico analysis. Sci Rep. 2017;7(1):8612. doi:10.1038/s41598-017-08986-9

10. Aytac Z, Uyar T. Core-shell nanofibers of curcumin/cyclodextrin inclusion complex and polylactic acid: enhanced water solubility and slow release of curcumin. Int J Pharm. 2017;518(1-2):177-184. doi:10.1016/j.ijpharm.2016.12.061

11. Luong D, Kesharwani P, Alsaab HO, et al. Folic acid conjugated polymeric micelles loaded with a curcumin difluorinated analog for targeting cervical and ovarian cancers. Colloids Surf., B. 2017;157:490-502. doi:10.1016/j.colsurfb.2017.06.025

12. Rizvi SA, Saleh AM. Applications of nanoparticle systems in drug delivery technology. Saudi Pharma J. 2018;26(1):64-70. doi:10. 1016/j.jsps.2017.10.012

13. Ahmad N, Ahmad I, Umar S, Iqbal Z, Samim M, Ahmad FJ. PNIPAM nanoparticles for targeted and enhanced nose-to-brain delivery of curcuminoids: UPLC/ESI-Q-ToF-MS/MS-based pharmacokinetics and pharmacodynamic evaluation in cerebral ischemia model. Drug Deliv. 2016;23(7):2095-2114. doi:10.3109/10717544. 2014.941076
14. Reddy B, Yadav HK, Nagesha DK, Raizaday A, Karim A. Polymeric micelles as novel carriers for poorly soluble drugs. J Nanosci Nanotechnol. 2015;15(6):4009-4018. doi:10.1166/jnn.2015.9713

15. Faiyazuddin M, Ahmad N, Khar RK, Bhatnagar A, Ahmad FJ. Stabilized terbutaline submicron drug aerosol for deep lungs deposition: drug assay, pulmonokinetics and biodistribution by UHPLC/ ESI-q-TOF-MS method. Int J Pharm. 2012;434(1-2):59-69. doi:10. 1016/j.ijpharm.2012.05.007

16. Rauf A, Ahmad N, Mirza MA, et al. Development and in vitro evaluation of budesonide submicron drug particles: an approach to improve lungs deposition and dissolution for pulmonary delivery. J Aerosol Med Pulm Drug Deliv. 2013;26(4):A255.

17. Chen S, Han Y, Sun C, et al. Effect of molecular weight of hyaluronan on zein-based nanoparticles: fabrication, structural characterization and delivery of curcumin. Carbohydr Polym. 2018;201:599-607. doi:10.1016/j.carbpol.2018.08.116

18. Chen S, Han Y, Huang J, et al. Fabrication and characterization of layer-by-layer composite nanoparticles based on zein and hyaluronic acid for codelivery of curcumin and quercetagetin. ACS Appl Mater Interfaces. 2019;11(18):16922-16933. doi:10.1021/acsami. 9 b02529

19. Allijn IE, Schiffelers RM, Storm G. Comparison of pharmaceutical nanoformulations for curcumin: enhancement of aqueous solubility and carrier retention. Int J Pharm. 2016;506(1-2):407-413. doi:10. 1016/j.ijpharm.2016.04.070

20. Cooksey TJ, Singh A, Le KM, et al. Tuning biocompatible block copolymer micelles by varying solvent composition: core/corona structure and solvent uptake. Macromolecules. 2017;50 (11):4322-4334. doi:10.1021/acs.macromol.6b02580

21. Lin R, Cui H. Supramolecular nanostructures as drug carriers. Curr Opin Chem Eng. 2015;7:75-83. doi:10.1016/j.coche.2014.11.005

22. Cuomo F, Perugini L, Marconi E, Messia MC, Lopez F. Enhanced curcumin bioavailability through nonionic surfactant/caseinate mixed nanoemulsions. J Food Sci. 2019;84:2584-2591. doi:10.1111/jfds.v84.9

23. Feng J, Wu S, Wang H, Liu S. Stability of trianionic curcumin enhanced by gemini alkyl O-glucosides and alkyl trimethyl ammonium halides mixed micelles. Colloids Surf Physicochem Eng Asp. 2016;504:190-200. doi:10.1016/j.colsurfa.2016.05.076

24. Bar-Zeev M, Nativ L, Assaraf YG, Livney YD. Re-assembled casein micelles for oral delivery of chemotherapeutic combinations to overcome multidrug resistance in gastric cancer. J Mol Clin Med. 2018;1 (1):55-65.

25. El-Far SW, Helmy MW, Khattab SN, Bekhit AA, Hussein AA, Elzoghby AO. Phytosomal bilayer-enveloped casein micelles for codelivery of monascus yellow pigments and resveratrol to breast cancer. Nanomedicine. 2018;13(5):481-499. doi:10.2217/nnm-2017-0301

26. Głąb TK, Boratyński J. Potential of casein as a carrier for biologically active agents. Top Curr Chem. 2017;375(4):71. doi:10.1007/ s41061-017-0158-z

27. Konekeri V, Bennadi D, Manjunath M, Kshetrimayum N, Siluvai S, Reddy CVK. A clinical study to assess the effectiveness of CPP-ACP (casein phosphopeptide-amorphous calcium phosphate) versus potassium-nitrate (KNO3) on cervical dentine hypersensitivity. J Young Pharm. 2015;7(3):217. doi:10.5530/jyp.2015.3.12

28. Bagchi D, Chaudhuri S, Sardar S, et al. Modulation of stability and functionality of a phyto-antioxidant by weakly interacting metal ions: curcumin in aqueous solution. RSC Adv. 2015;5(124):102516102524. doi:10.1039/C5RA21593E

29. Kharat M, Du Z, Zhang G, McClements DJ. Physical and chemical stability of curcumin in aqueous solutions and emulsions: impact of $\mathrm{pH}$, temperature, and molecular environment. J Agric Food Chem. 2017;65(8):1525-1532. doi:10.1021/acs.jafc.6b04815

30. Li J, Lee IW, Shin GH, Chen X, Park HJ. Curcumin-eudragit ${ }^{\circledR}$ E PO solid dispersion: a simple and potent method to solve the problems of curcumin. Eur J Pharm Biopharm. 2015;94:322-332. doi:10.1016/j. ejpb.2015.06.002 
31. Fan N, Ma P, Wang X, et al. Storage stability and solubilization ability of HPMC in curcumin amorphous solid dispersions formulated by eudragit E100. Carbohydr Polym. 2018;199:492-498. doi:10.1016/j.carbpol.2018.07.036

32. Wang S, Ye F, Wei F, Zhao G. Spray-drying of curcumin-loaded octenylsuccinated corn dextrin micelles stabilized with maltodextrin. Powder Technol. 2017;307:56-62. doi:10.1016/j.powtec.2016.11.018

33. Cho H, Kim B, Chun J, Choi M. Effect of spray-drying process on physical properties of sodium chloride/maltodextrin complexes. Powder Technol. 2015;277:141-146. doi:10.1016/j.powtec.2015.02. 027

34. Cicerone MT, Pikal MJ, Qian KK. Stabilization of proteins in solid form. Adv Drug Deliv Rev. 2015;93:14-24. doi:10.1016/j.addr.20 15.05 .006

35. Devineni D, Gonschorek C, Cicerone MT, Xu Y, Carpenter JF, Randolph TW. Storage stability of keratinocyte growth factor-2 in lyophilized formulations: effects of formulation physical properties and protein fraction at the solid-air interface. Eur J Pharm Biopharm. 2014;88(2):332-341. doi:10.1016/j.ejpb.2014.05.012

36. Xu Y, Grobelny P, Von Allmen A, et al. Protein quantity on the airsolid interface determines degradation rates of human growth hormone in lyophilized samples. J Pharm Sci. 2014;103(5):1356-1366. doi:10.1002/jps.23926

37. Yusuf H, Nugraheni RW, Setyawan D. Effect of cellulose derivative matrix and oligosaccharide on the solid state and physical characteristics of dimethyldioctadecylammoniumliposomes for vaccine. Res Pharm Sci. 2019;14(1):1. doi:10.4103/1735-5362.251847

38. Priya P, Raj RM, Vasanthakumar V, Raj V. Curcumin-loaded layer-by-layer folic acid and casein coated carboxymethyl cellulose/ casein nanogels for treatment of skin cancer. Arabian J Chem. 2017;13(1):694-708.
39. Fan N, He Z, Ma P, et al. Impact of HPMC on inhibiting crystallization and improving permeability of curcumin amorphous solid dispersions. Carbohydr Polym. 2018;181:543-550. doi:10.1016/j. carbpol.2017.12.004

40. Har CL, Fu N, Chan ES, Tey BT, Chen XD. In situ crystallization kinetics and behavior of mannitol during droplet drying. Chem Eng J. 2018;354:314-326. doi:10.1016/j.cej.2018.07.202

41. Nugraheni RW, Setyawan D, Yusuf H. Physical characteristics of liposomal formulation dispersed in HPMC matrix and freeze-dried using maltodextrin and mannitol as lyoprotectant. PHARM. 2017;23 (4):285-292.

42. Ye R, Cheng Q, Cai J, Feng T, Wang G. Stable casein-hydroxypropyl cellulose complexes at low pH. J Food Qual. 2016;39(4):292-300. doi:10.1111/jfq.2016.39.issue-4

43. Jagtap S, Magdum C, Jadge D, Jagtap R. Solubility enhancement technique: a review. Int J Pharm Sci Res Int. 2018;10(9):2205-2211.

44. Marano S, Barker SA, Raimi-Abraham BT, Missaghi S, RajabiSiahboomi A, Craig DQ. Development of micro-fibrous solid dispersions of poorly water-soluble drugs in sucrose using temperature-controlled centrifugal spinning. Eur J Pharm Biopharm. 2016;103:84-94. doi:10.1016/j.ejpb.2016.03.021

45. Madgulkar A, Bandivadekar M, Shid T, Rao S. Sugars as solid dispersion carrier to improve solubility and dissolution of the BCS class II drug: clotrimazole. Drug Dev Ind Pharm. 2016;42(1):28-38. doi:10.3109/03639045.2015.1024683

46. Fujimori M, Kadota K, Shimono K, Shirakawa Y, Sato H, Tozuka Y. Enhanced solubility of quercetin by forming composite particles with transglycosylated materials. $J$ Food Eng. 2015;149:248-254. doi:10.1016/j.jfoodeng.2014.10.010
International Journal of Nanomedicine

\section{Publish your work in this journal}

The International Journal of Nanomedicine is an international, peerreviewed journal focusing on the application of nanotechnology in diagnostics, therapeutics, and drug delivery systems throughout the biomedical field. This journal is indexed on PubMed Central, MedLine, CAS, SciSearch ${ }^{\mathbb{}}$, Current Contents ${ }^{\mathbb{R}} /$ Clinical Medicine,

\section{Dovepress}

Journal Citation Reports/Science Edition, EMBase, Scopus and the Elsevier Bibliographic databases. The manuscript management system is completely online and includes a very quick and fair peer-review system, which is all easy to use. Visit http://www.dovepress.com/ testimonials.php to read real quotes from published authors. 